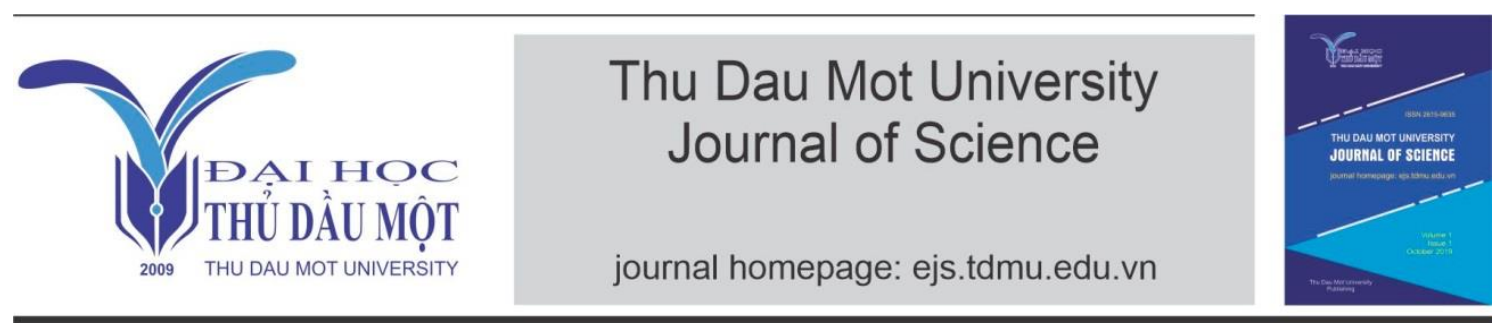

\title{
A study about the sustainable challenges of extended areas in Taiwan and lessons for Ho Chi Minh, Dong Nai and Binh Duong regions
}

\author{
by Tran Dinh Hieu (Thu Dau Mot University), Nguyen Hong Giang ( National \\ Kaoshiung University of Science and Technology of Taiwan)
}

\begin{abstract}
Article Info: $\quad$ Received 2 Oct. 2020, Accepted 10 Dec 2020, Available online 15 Dec, 2020
Corresponding author: hieutd@tdmu.edu.vn; gianghueuni@gmail.com

https://doi.org/10.37550/tdmu.EJS/2020.04.085
\end{abstract}

\begin{abstract}
This paper examines the main challenges of the processes of space and social policy change present to current urbanization trends of Taiwan. The chapter argues that one of the main challenges is economic growth, increasing integration into the global economy and making Taiwan competitive in the global economy. This process leads to the growth of large urban regions that present many challenges to the urban development in the future. In particular, the paper focuses on the most fragile areas of the extended urban spaces are the rural and urban margins, where urban activities are expanding into densely populated agricultural regions. It is argued that in these areas, local policies should be developed that adapt to local ecosystems. The paper presents lessons of interventions in this field for Ho Chi Minh, Dong Nai and Binh Duong Region for urban expansion.
\end{abstract}

Keywords: southeast area, triangular area

\section{Introduction}

The speed of urbanization is growing strongly in the 21 st century, and urbanization has raised a number of problems related to the expansion of urban areas. The areas that are expanded in these urban areas are usually rural areas and large mountainous areas. These areas face problems such as a lack of energy economy and a declining population 
as young people move into big cities to work (McGee, 2017). However, the areas that have not been built are much, they have great potential for long-term urbanization models. (Pawson, 2008; McGee, 1991). (Pawson, 2008; McGee, 1991)

Expansion of urban space is the widespread area of urban activities taking place at the edge of urban areas in the overall urban space. This is not only at the level of the administratively defined urban area, but also at the urban core level with the expansion of urban space. This expansion is most commonly occurring in large cities and smaller cities in the urban hierarchy. These expanded regions are also the locations that generate increasing national GDP. (McGee, 2017)

Spatial expansion of urban areas in Taiwan to focus population in urban areas, especially in large cities in the north and west of the country (Huang, 2019). Expansion of urban space is often the growth model in important ways that are substantially different from those of other developed countries. At the same time, ensure the longterm environmental sustainability of the island's major regions. However, applying an urbanization experience from one country to another is a very careful task, as local conditions and historical institutions are very important in influencing the results. The most important factors of Taiwan's interest are the extreme fragmentation of land ownership, the political power of farmers, and the extremely low tax rates for agricultural land in urban areas (Cheng, 1990).

This paper examines the expansion of adjacent urban space among metropolitan areas in Taiwan, explores specific challenges for sustainable development due to urban space expansion, and addresses ideas. The analysis of the spatial structure is most important implications for sustainable policies in expanding urban space for the crowded triangular areas of Ho Chi Minh City, Dong Nai and Binh Duong Province.

\section{Taiwan extended urban space}

Expanding urban space to meet the main development trend and solve the problems facing spatial development. Expanding space to conserve land and sustainably such as developing industrial economy, sustainable development of urban areas, transportation and ensuring the life of urban people. Taiwanese government has planned this country into four levels: International level, National level, Regional level and Local level, Liaw \& Chiang, 2014).

2.1. International Level: In world networks, Taiwan makes up important positions as a key node in the domains of ICT R\&D and manufacturing, S\&T innovation, agricultural technologies, Chinese culture, tourism, and an Asia-Pacific logistics gateway.

2.2. National Level: Hierarchy of the level includes Central Mountain Range conservation axis, Innovation development axis in Western of Taiwan, prime living and industrial axis, in Eastern of Taiwan Sea belt Eco-tourism areas on offshore islands. 

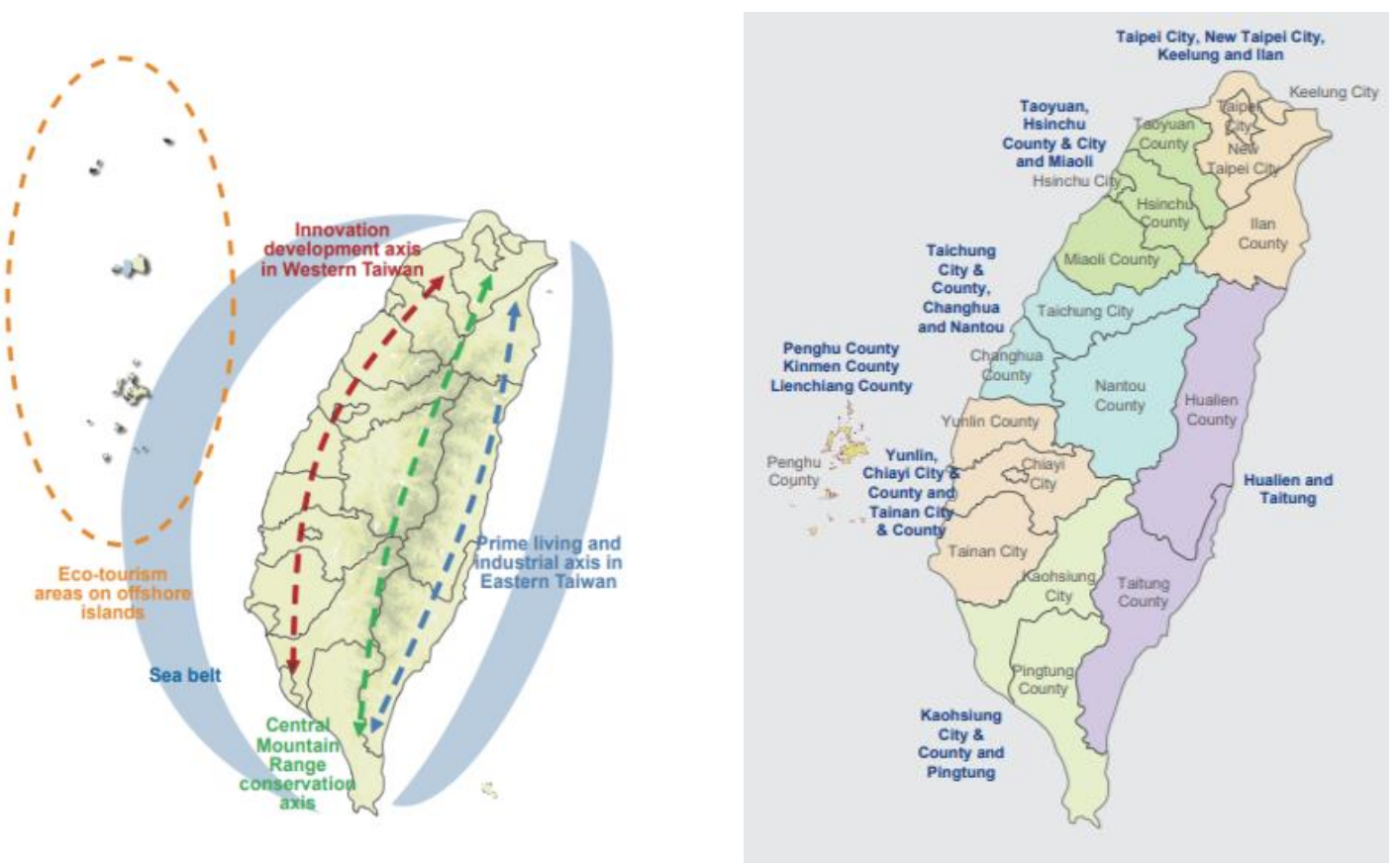

Figure1. National spatial structure by regional level: Seven regional living circles (source:Strategic plan for national spatial development-summary, Tai-Jian-Zi-No.099002926)

- Central Mountain Range conservation axis: This axis mainly be focused on ecological conservation and protecting indigenous people's culture.

- Innovation development axis in Western of Taiwan: This is creativity and innovation axis to serve as the core values for urban-rural transformation and development in Western of Taiwan.

- Prime living and industrial axis in Eastern of Taiwan: The aim of the axis is to further blend new lifestyle concepts such as lifestyles of health and sustainability (LOHAS), slow living, and health-enhancing recreation into the region's industry, to form a development model that, differing from the development model for Western of Taiwan. Effectively taps into the Eastern region's rich and diverse cultural features, slow tempo of life, beautiful natural scenery, clean land resources, and other advantageous conditions, to develop the region.

- A sea belt: The purpose of the axis is to actively pursue R\&D and applications in respect of ocean thermal energy conversion, seawater extraction, and various areas of biotechnology.

- Offshore islands: The main aim is to place on environmental conservation and cultural preservation spaces, with the development of unique ecological and cultural experiences. 
2.3.Regional Level: There are three major city-regions and Eastern region that is Northern city-region, Central city-region, Southern city-region, and Eastern region.
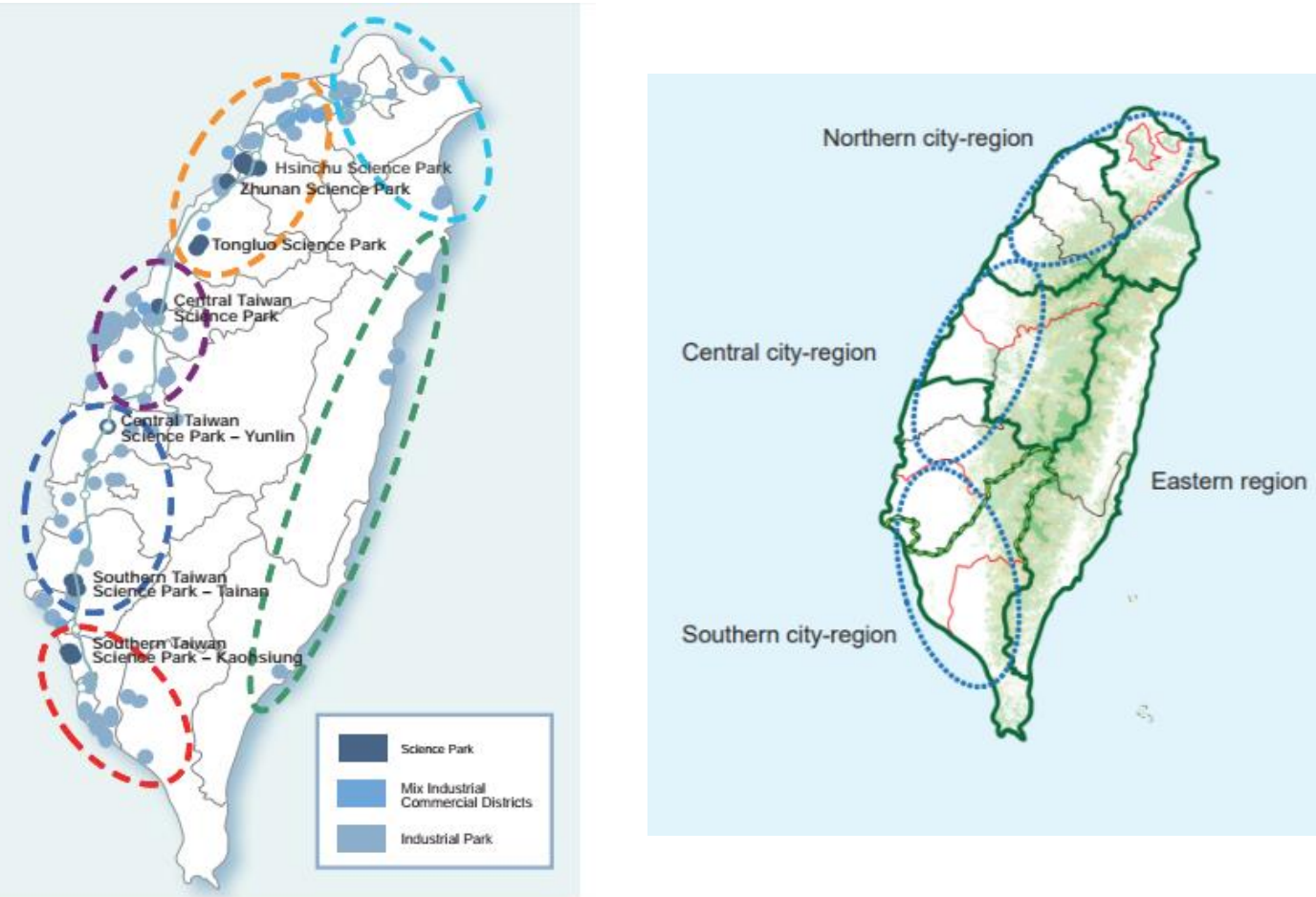

Figure 2. National spatial structure by regional level: Three major city-regions and Eastern region

(source: Strategic plan for national spatial development-summary, Tai-Jian-Zi-No.099002926)

- The Northern city-region: The main function is to place on accentuating its unique strengths as the basis for differentiating it from regional rivals, to highlight its distinctiveness and present its international competitiveness.

- The Central city-region: The aim is to bolster the development of innovative industries related to living and leisure.

- The Southern city-region: creativity, digital software, and waterside tourism, linking Kaohsiung Port with the rising ports of southeastern mainland China, to form a group of competing and cooperating ports to support the formation of industrial chains across the Taiwan Strait.

- The Eastern city-region: The aim for the axis is as same of prime living and industrial axis in the Eastern of Taiwan

2.4.Local Level: The level includes seven regional living circles and city-county cooperation regions i.e. Taipei City, Taipei County (Xinbei City, New Taipei City), Keelung City and Ilan; Taoyuan, Hsinchu and Miaoli; Taichung, Changhua and Nantou; 
Yunlin, Chiayi and Tainan; Kaohsiung City, Kaohsiung County and Pingtung; Hualien and Taitung; Penghu, Kinmen and Matsu. Cross-regional platforms of city-county cooperation regions.

The aim is that each of the regional living circles will encompass sufficient population and hinterland to support its development and consumer markets. The division enable greater economic effects to be gained from infrastructural investment. Each city can establish its own status and create its own distinctive competitive advantages in accordance with its particular industrial and geo-environmental characteristics.

\section{The issues of extended urban space}

The levels of extended urban development described in the previous section have broad implications for the short and long term sustainability of Taiwan's cities. The extended urban space is valuable in that it is urbanization of high-density rural areas, which are relatively easily and quickly integrated into metropolitan economies and provide a fertile medium for economic growth (Sorensen, 2009). However, expanding urban space also has some advantages and disadvantages as follows:

\subsection{Advantages}

- Extended urban space consolidates urban economic growth over large, semi-rural regions advantageous for rapid economic development. The integration of enormous areas adjacent and between urban centers into urban economic structures provide a large and inexpensive workforce, which owns its own land and housing, maintains traditional community social support structures.

- Enormous land areas also become available for new factory and housing use, with little need for basic infrastructure, as traditional means of solid and liquid waste disposal continue to function. At the same time, the old rural road system can allow greatly increased mobility with the spread of two-stroke motorcycles and scooters.

\subsection{Disadvantages}

- Implementation mechanisms of extended urban space are not well monitored by strict control of an equivalent level of government, and policies are repeatedly debated and their details manipulated this way and that, it will have a minimal formalistic effect. For instance, environmental protection and the petrochemical industry over issues involving micro and macro perspectives, such as procedures for changing land use (urban or non-urban planning), environmental impact assessment review, the timely supply of water and electricity resources, external roadways, and other ancillary measures, it is essential to have a higher level of guiding principles to refer to in making policy decisions. 
- Prioritization of budget execution for urban development needs funds. Each year, at all government levels, funds are endowed through ordinary budgets, fund budgets, or special central government budgets, for major development projects with a bearing on national spatial development. Many development projects have been delayed due to fluctuations in material prices, environmental impact assessment reviews, and plan modification. Therefore, their budgets could not be executed smoothly, which has caused even greater squeezing of funds. In particular, local governments incessantly propose a great number of plans to vie for their development budgets. However, since these projects inevitably lack a cross-regional perspective, they result in the construction of similar facilities that end up having poor use efficiency and wasting resources.

- There is a relative lack of capacity for prompt response from a macro-developmental mentality and actions that government agencies and regulation present toward national spatial development. Consequently, government agencies form many internal committees or task forces that recruit the participation of outside members, which often creates a cramping effect on policy correspondence. At the same time, laws and regulations also become elastically fatigued and unable to respond to new trends of perspective.

\section{Lessons of the sustainable extended urban space for triangular areas of Ho Chi Minh City, Binh Duong and Dong Nai province.}

\subsection{The issue of land management in Taiwan}

Extended urban space present special problems and challenges for sustainable urban management in Taiwan, which are clearly the result of political and social factors to the island. The aspects of the development patterns peculiar to expanded urban space may be expected to present real obstacles to the creation of socially, fiscally and environmentally sustainable urbanization to others. In particular, the high densities of rural population before urbanization mean that growth management and the prevention of urban sprawl are extremely difficult. Urban fringe land development common in Taiwan support the basic arguments for land development controls, that such controls are essential to be able to protect the public welfare through the efficient and timely provision of local public space such as roads, sewers, and parks.

\subsection{The issues of land management in the triangle region of $\mathrm{Ho}$ Chi Minh City, Binh}

\section{Duong Province and Dong Nai province}

Although, enterprises ability to access land in Ho Chi Minh City is high, but it is still lower than other localities. In the area, only $88 \%$ of the land area has certificate of land use, lower than Binh Duong (98\%), Dong Nai province (93\%) and average level of the 
Mekong Delta zone (96\%). The proportion of enterprises has land of business using and certificates of land use is only 37\%, much lower more than Binh Duong province and average level of the Mekong Delta zone (about 70\%). In addition, the risk assessment of land acquisition of government is higher more than other localities. If acquired land will be adequately compensated in Ho Chi Minh City, which is evaluated lower than other localities in zone and only $25 \%$ of enterprises believe that, they will always or often be adequately compensated if they are acquired land. Otherwise, only $12 \%$ of non-state enterprises in Ho Chi Minh City are agreed that, they would not encounter obstacles in land accession or land area extension, much lower than Binh Duong province and Mekong Delta zone.

\subsection{From analysis on above and the existing situation in the study area, the author proposes five points that should be learned from the Taiwan experience of extended urban space.}

- First, a core issue is the rapid increase in land values across very large areas throughout the urban space expansion as they are integrated into the urban economy. Although it is unlikely that much of the land will be converted to urban use in a very long time to come, all landowners explain the reasonable expectation that their land, when put to urban uses and will be using values in city. Therefore, they are reluctant to sell unless they can do so at a high price, expecting even greater profits in the future. Expectations of potential for urban development have a host of negative impacts in the case of Taiwan, including strong land owners' resistance to development control regimes and the practice of developing a small plot of land to increase capital and set the level of urban value for the remainder on the spread of urban land prices across large rural areas, often decades before significant urbanization, also creates real problems for local governments accused of providing urban infrastructure, because public space like roads, parks and schools require a lot of land. However, in Taiwan, allowing relatively uncontrolled development throughout the extended urban space meant that urban land values were not a function of urban services, or a development permit, but simply of landowner expectations of future development potential.

- Second important lesson, as a country developing, the cost of building roads, sewers and subways can be increasingly affordable, even if the cost of buying land can be increasingly out of reach. Across the Taipei area, the main constraint in the provision of public space and urban finance is the current high land costs. The obvious conclusion is that securing land for public use is the most important goal early in the development process and expensive investments in less important base. Such investments can easily be made later, as capital and technological capacity become more abundant, especially if adequate land reserves have been established. 
- Third, important and environmentally sensitive areas such as along waterways, watersheds, wetlands and coastlines. These areas are essential for the protection of water resources and wildlife habitats.

- Fourth, the main natural areas are permanently abandoned, such as forests, wetlands and steep slopes. These areas, if developed into urban use, may cause environmental risks in the future. Such areas can also provide accessible green spaces for urban residents and help minimize urban heat island problems.

- Fifth, some areas of high yielding agricultural land are close to urban areas, so in many cases they need to be protected for long-term food supply.

- Final, corridors should be designated for future major transport infrastructure before urban development.

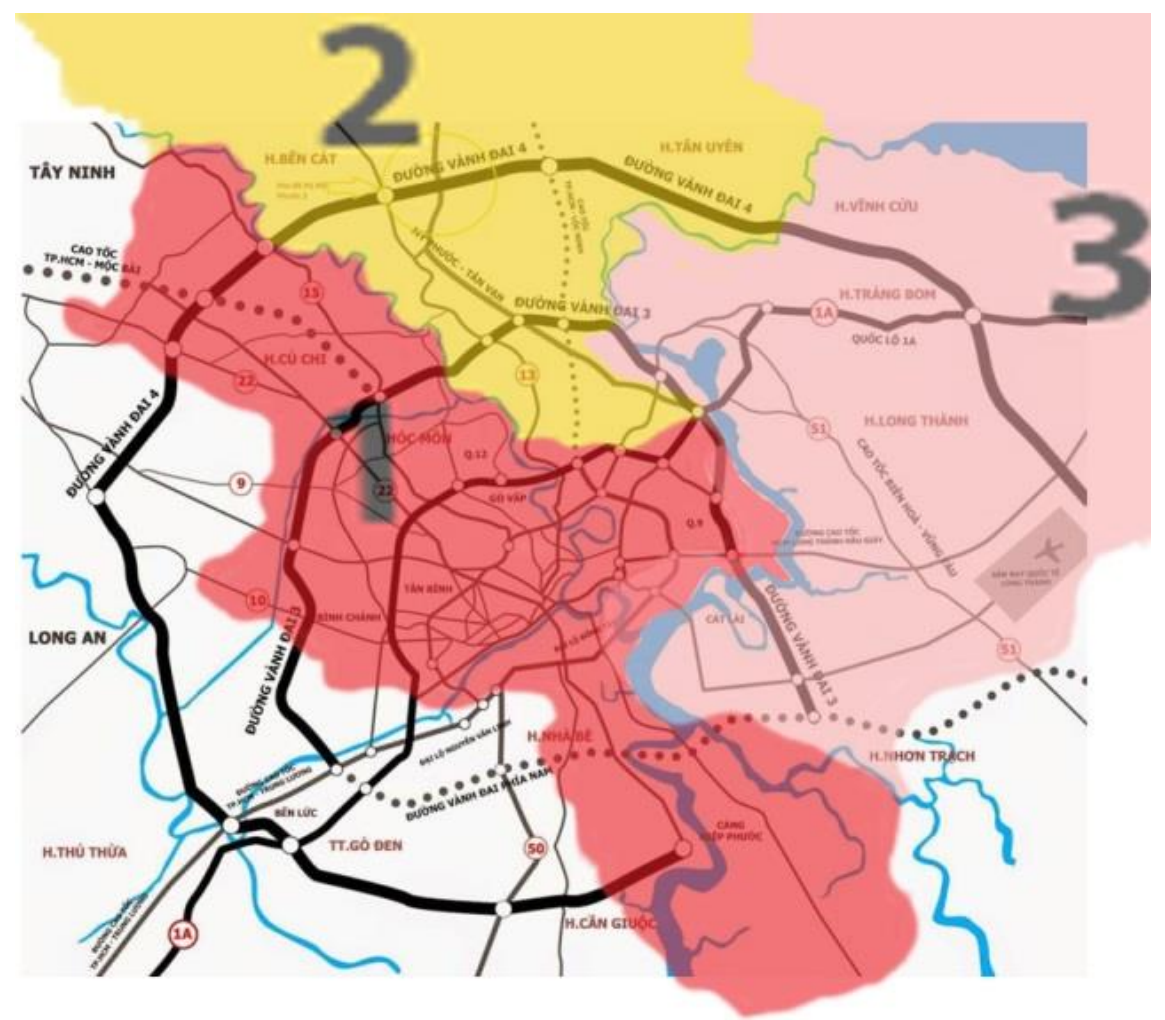

Figure 3. Transport system of the belt roads developing the triangle area

1. Ho Chi Minh (red colour); 2. Binh Duong (yellow colour); 3. Dong Nai (pink colour)

(Source: authors based on current planning status)

Ho Chi Minh City is well connected by road with provinces of Binh Duong, Dong Nai,... in the southern key economic region through a system of national highways such as national highway of $1 \mathrm{~A}, 1 \mathrm{~K}, 13,22 \mathrm{~A}$ (road is trans-asean), national highway 52 (Hanoi highway). belt roads 2, 3, 4 will be built in the future, will increase the connectivity of Ho Chi Minh City with major roads system in the region. (Fig. 3). 


\subsection{Comments}

Ho Chi Minh City, Binh Duong and Dong Nai Province are among the most development triangle areas in Vietnam and the urbanization rate of this area is increasing rapidly. However, urbanization to meet the needs of population growth will lead to a number of problems arising between areas such as: urban areas and rural areas, areas of bordering between provinces in the future. Therefore, the lessons of urban expansion in Taiwan have some meaning and to should be referenced (as above), that to help planning for the development of this triangular areas.

\section{Conclusion}

Expansion of urban space is a large area of urban activities that takes place at the edge of urban areas in urban space in general. This is not only at the level of the administratively defined urban area, but also at the urban core level with the expansion of urban space. Taiwan is ahead of the decades of other Asian developing countries in the process of economic development so Taiwan's experience can serve, if not a prediction of urban issues in future. The experiences of expanding urban space for industrial development in urban areas are experience lessons for urban development for the dynamic triangle areas of Ho Chi Minh City, Binh Duong and Dong Nai province.

\section{Reference}

Cheng, T.-j. (1990). Political regimes and development strategies: South Korea and Taiwan. Manufacturing miracles: paths of industrialization in Latin America and East Asia, 139178.

Huang, W.-J. (2019). The New Spatial Planning Act in Taiwan: A messy shift from economic development-oriented planning to environmental conservation-oriented planning? Planning practice \& research, 34(1), 120-130.

Liaw, C.-H., \& Chiang, Y.-C. (2014). Dimensionless analysis for designing domestic rainwater harvesting systems at the regional level in northern Taiwan. Water, 6(12), 3913-3933.

McGee, T. G. (2017). The Sustainability of Extended Urban Spaces in Asia in the Twenty-First Century: Policy and Research Challenges. In Sustainable Landscape Planning in Selected Urban Regions (pp. 17-26): Springer.

Sorensen, A. (2009). Megalopolitan Development and the Transformation of Rural Japan: Sustainability Implications of Extended Metropolitan Regions in Asia. Human Settlement Development-Volume I, 186. 